MODELING, IDENTIFICATION AND CONTROL, 1996, VOL. 17, NO. 1, 63-73

doi:10.4173/mic.1996.16

\title{
Ideas for underwater $3 D$ sonar range sensing and environmental modeling
}

\author{
P. G. AURAN $\dagger$ and O. SILVEN $\ddagger$
}

Keywords. 3D sonar range sensing, data representation.

\begin{abstract}
A 3D spatial grid for exploiting the range and direction information inherent in sonar range data is presented. Special attention is given to the realtime performance of this representation, i.e. it should be a feasible way of representing the 3D range data acquired by an operating AUV.
\end{abstract}

\section{Introduction}

Intelligent vehicles will play a major role in future underwater missions, and independent and meaningful interpretation of sensor data is a principal prerequisite for accomplishing high-level autonomous underwater (AUV) missions (Brutzmann, Compton and Kanayama 1992). Specially important is interpretation of sonar range data, as the sonar is one of the most useful sensors for underwater geometric exploration. Much work has already been reported on sonar vision processing, some examples could be found in (Brutzmann, Compton and Kanayama 1992; Elfes 1990; Elfes 1991; Leonard, Durrant-Whyte and Cox 1992; Mandelbaum and Mintz 1994; Mathisen 1993; Matthies and Elfes 1988; Moran, Leonard and Chryssostomidis 1993; Smith, Self and Cheeseman 1987). Most of the work is however done in two dimensions, i.e. it addresses the construction of 2D cross-sectional geometrical models or environment maps. For the purpose of environmental modeling by using line and arch primitives (Mathisen 1993), it is assumed the object of interest has a constant cross-sectional geometry, an assumption maybe more suitable for controllable scenes of man-made objects than for a 'real-world' operating AUV. The AUV's perception process must be able to cope with sonar input describing arbitrary shapes.

Of special interest is the sonar research reported by Elfes (Matthies and Elfes 1988; Elfes 1990; Elfes 1991) presenting the occupancy grid framework. The occupancy grid is a spatial map of the environment, consisting of small cells which either is 'empty' or 'occupied'. Each cell's state is estimated a Bayesian stochastic approach. Elfes (Elfes 1990) concludes that the occupancy grid framework could be used for integration of multisensor data (for a reference to this principle, see Durrant-Whyte (1988)). This model is suitable for realtime obstacle avoidance, mobile robot mapping and navigation, and it supports robust sensor interpretation methods, incremental discovery procedures and explicit handling of uncertainty (Elfes 1990).

The reported research is concerned with building 2D-maps using ultrasonic (and stereo vision) sensors operating in air, i.e. the issue is obstacle avoidance and path

Received 20 August 1995.

† University of Trondheim, The Norwegian Institute of Technology, Department of Engineering Cybernetics, N-7034 Trondheim, Norway (E-mail:pga@itk.unit.no).

†University of Oulu, Department of Electrical Engineering, SF-90570 Oulu, Finland.

Presented at the 3rd IFAC Workshop on Control Applications in Marine Systems, May 1995, Trondheim, Norway (CAMS '95) 
planning of mobile robots operating in 'office-like' environments. With the operation of a 6 DOF (degree of freedom) AUV in mind, some remarks ought to be made:

- The ultrasonic range transducers have different properties when compared to marine sonars, i.e. broader beam and shorter range. Sensor range compared to vehicle size is not comparable with the AUV case, which will affect the objectives of sonar perception and navigation.

- Although well-known, the significant different characteristics of sound propagation in air compared to water should be pin-pointed. Effects like signal damping, background noise, temperature gradients (Ol'shevskii 1978; Urick 1983) are not recognized by experiments in air. The conclusions drawn from such experiments will be limited for the AUV-case.

- The proposed Bayesian cell updating approach mentioned in Elfes (1990) demands an assumption of independent cell states to be feasible. It also constitutes an extensive cell updating scheme as all cells in the map must be updated as new information enters the occupancy grid. In a realtime perspective this is certainly only feasible with a limited number of cells. In 3D these remarks are of vital importance for the feasibility of the occupancy grid approach.

- In the proposed 2D environmental maps, the cell size is quite coarse (comparable to sensor resolution at maximum range), and also fixed, e.g. not exploiting the resolution properties of the sensor (range accuracy varies with distance). If the map is not intended for extracting geometric properties, this may not be of significant importance.

Traditionally sonar data are presented as polar plots (range and bearing) for human interpretation. As sonar range data in nature are three-dimensional, it should be interpreted as such by the AUV. Taking into account the plane of sweep (for a narrowband sweep sonar) or the 'beam-plane' (for a multibeam sonar), the bearing gives information in two dimensions, i.e. the two angles in a spherical coordinate system.

In this article the sonar is regarded as a range sensor with certain geometric and uncertainty features. More detailed modeling of underwater echo-ranging requires complicated solutions of the wave equation in a non-homogeneous medium, which is outside the scope of this article. The main objective here is range data organization, uncertainty modeling and interpretation of stationary objects for purposes like environmental mapping, obstacle avoidance and path planning.

A spatial framework for the exploitation and active use of the 3D sonar range data is presented. An important aspect of this approach is the concept of active vision (Bajcsy 1988). The presented ideas are highly motivated by the occupancy grid approach. A 3D occupancy grid is used to effectively organize actively sensed sonar range data. This grid could be interpreted as a local $3 D$ map of sensor information useful for accomplishing the AUV's current task.

\section{Overview}

The main objective of the grid will be as a first order modeling process for AUV-relative navigation and world sensing. By this is meant how to perform path planning and inspection using sonar ranging from the AUV's point of view. One actual problem could be to plan navigation for 'getting a closer look', possibly utilizing other sensors as well (for example using video camera at short ranges). Assuming the AUV to operate in 'expedition or exploration mode', the grid is a first order model of what 


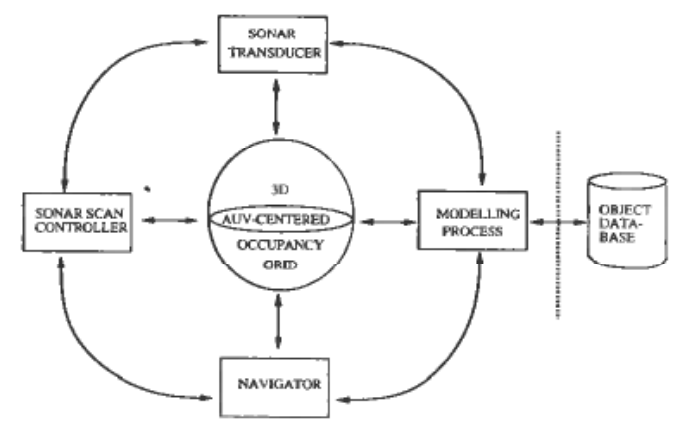

Figure 1. The 3D grid at the AUV's core for world perception.

the AUV senses from the current position integrated with that it has sensed on its way to this position. The 3D grid is therefore AUV-centred.

At this point it should be noted that the grid is not meant to be the only world model, but a 'first' model, a fast organization of raw input data of current interest. The AUV will depend on multi-sensor input to be able to execute its task satisfactorily and the 3D grid should therefore be seen in this perspective. The 3D grid could be used as a core of organized sonar data by several processes in the AUV's expert system, as outlined in Fig. 1. It could be interfaced with a 'Navigator' process including a vessel dynamics model, as found in Fossen (1991), or with a bathymetric map positioning system with an inertial navigation system (INS) as presented in (Bergman, Andersen and Christensen 1993, Bergem 1993a, Bergem 1993b). A control process for sonar scan directions (sweep planes and beam configuration) could also be included in the expert system. Furthermore a 'Modeling' process is suggested for extracting high-level geometric information, possibly also utilizing object recognition by using an object database. All these processes are running in parallel and interacting in a complex AUV expert system, similar to ideas mentioned in Brutzmann, Kanayama and Zyda (1992). The 3D grid would be an organized core of useful information in this expert system.

\section{Motivation for a 3D occupancy grid}

\subsection{Some features of $2 D$ echo ranging}

A lot of research has been done on 2D geometric modeling, and it is therefore natural to summarize some features of $2 \mathrm{D}$ sonar ranging:

- Regions of constant depth (RCDs) are important features useful for separating echoes from different objects, and also for filtering multipath echoes (Leonard, Durrant-Whyte and Cox 1992).

- Tangential clusters (Mandelbaum and Mintz 1994), or echoes with similar tangent direction, could be used to detect planar surfaces and corners. This principle is suggested for 3D, but experiments are in 2D.

- In Mathisen (1993) a methodology based on least squares fitting of line and arch primitives, and then producing hypotheses of objects or basic elements. It includes a parameter called 'accounted for' equal to the number of echoes each object is based on. The amount of echoes and also their repetitions indicates strong target features. For fixed objects echo stationarity is another important feature.

- The effect that sonar echoes near corners poorly describes their location should he acknowledged (Brutzmann, Compton and Kanayama 1992, Mathisen 1993). 
- When the environment includes machinery, for example in an underwater oil production scenario, passive sensing could be used to detect these objects. Rotating machinery could for example be identified using their resonant frequencies. This article addresses active and controlled sensing, and passive sonar sensing will not be further considered here.

\subsection{Active sonar sensing}

By active sensing it is understood that the sonar range data should be acquired in a controlled manner. Less sensing should be spent in areas of little interest, whereas sensing at objects and their characteristic features should be more thorough (Bajcsy 1988, Elfes 1990). Figure 2 shows the AUV coordinate system (top part of figure) and some important sonar parameters that could be dynamically controlled to utilize active sonar sensing with either a multibeam sonar or a narrow-beam sweep sonar (bottom part of figure). This active sensing approach is illustrated by the following principal $2 \mathrm{D}$
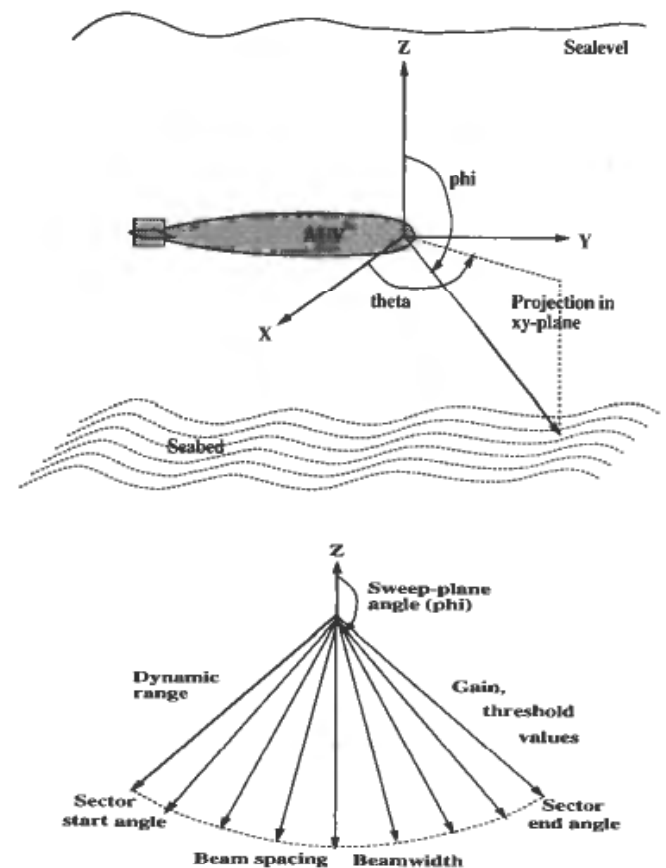

Figure 2. AUV coordinate frame and some controllable sonar parameters.

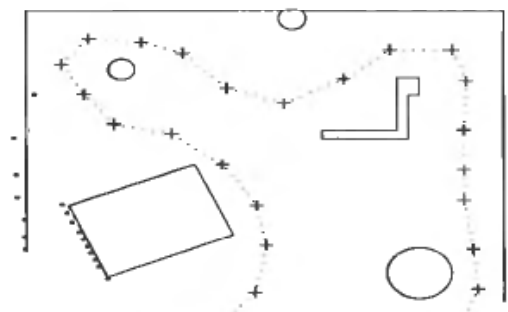

Figure 3. 2D geometric model and vehicle path. 
sketch. Figure 3 illustrates a 2D geometric environment model consisting of lines and circles. Inside this model a path is stipulated, with ' +' signs indicating the positions where sonar range data are obtained $\left(360^{\circ} \mathrm{scan}\right.$, limited sonar range). Noisy echoes from two of the edges, obtained at the first position in the vehicle path, are given in the figure. Assuming that the vehicle advances to the next position in the path, it will also receive echoes from a third edge (second lower edge on the parallelogram). The vehicle may now form a corner hypothesis, for example by a parametric regression scheme (Brutzmann, Compton and Kanayama 1992). At the two next positions it is possible to verify the corner hypothesis by observing that the echoes of the first edge disappears as one moves round the object. In this way the dynamic behaviour of echoes could be used as 'items of evidence'. If the vehicle movement and sonar scan directions are controlled to search for and exploit such events, the sensing will be active. Note also that events of this type could provide useful position information for increasing the navigation accuracy (assuming a priori knowledge of the scene). This is specially interesting if the AUV is equipped with an inertial navigation system where position errors increase by time (gyro drift problems).

\subsection{Grid representation}

The grid is a 3D sonar range 'image' of the AUV's close environment, i.e. the environment within the sonar range, a '3D wandering window' view of the world. Figure 4 illustrates a spherical versus a rectangular 'slice' of this window. Considering the characteristics of sonar data, it is clear that this 'window' should have certain properties:

- Sonar range echoes must be organized in a way that preserves the range and direction information, i.e. it should be easy and fast to check whether movement in a given direction is feasible or not.

- The grid should reflect the accuracy of the sonar range readings, i.e. the occupancy cells should not be too large. This is specially important for a modeling process using the grid as input data for extracting geometric information. Sensor characteristics should affect the grid configuration.
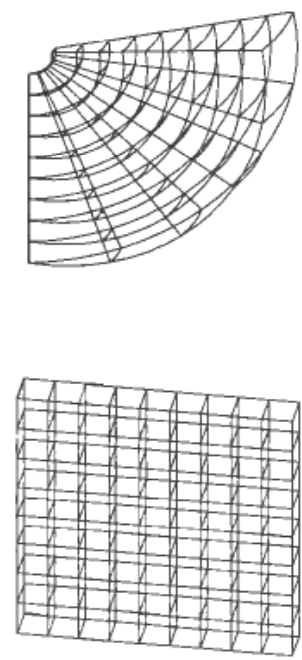

Figure 4. Spherical versus rectangular grid organization. 
- 'Curse of dimensionality': In 3D a fixed size rectangular array preserving sonar accuracy would be computer memory demanding, and grid updating time consuming. In contrast to the approach in Elfes $(1990,1991)$, one must focus on those cells that should be affected due to nearby echoes, and concentrate the amount of processing towards those (according to the principle of active perception). As sonar echoes in nature are sparse, this could be a feasible way of realizing a 3D occupancy grid, allowing reasonable update rates and computer memory demands.

\section{Simulations}

\subsection{The sonar sensor model}

As this point some early simulations within this framework has been done. A 2D sonar simulation model based on experiments from a Mesotech sonar (Mathisen 1993) was used to generate sonar-like data. This model is basically a $2 \mathrm{D}$ ray-tracing program that accepts an arbitrary input geometry consisting of arch and line segments. The rays are undersampled to a corresponding sonar beamwidth ( $2 \cdot 5$ degrees) and Gaussian noise is added according to a model similar to the one found in Elfes (1990):

$$
p((r, \theta) \mid(R, \Theta))=\frac{1}{2 \pi d R d \Theta} \exp ^{-\frac{1}{2}\left(\frac{(r-R)^{2}}{d R^{2}}+\frac{(\theta-\Theta)^{2}}{d \Theta^{2}}\right)}
$$

This 2D polar Gaussian probability model expresses the probability of an echo at one point $(r, \theta)$ given a sonar range reading at another one $(R, \Theta)$, assuming independent errors in radial and angular coordinates. The standard deviations ( $d R$ and $d \Theta)$ are given by the sonar sensor characteristics. Note that $d R$ is a function of range, typically given as a percentage of distance.

For 3D simulation the sonar simulation program is used to produce sonar-like echoes from cross-sections at different $z$-cordinates. When mapping sonar echoes to grid cells, the probability model in equation (1) is extended with another angular parameter of equal size (assuming conical beams). This model is used to map sonar range readings into grid cell states. Currently a simple scheme using cell midpoints and maximal responses has been implemented. More sophisticated algorithms for the grid updating process will be addressed when dealing with real sonar data.

Not only cells with actual sonar responses in them should be stored in the grid. A 3D uncertainty 'neighbourhood' centred at the physical echo should be affected, i.e. some sort of relaxation scheme could be useful. Some experiments with 3D masks related to the Gaussian noise model has been done, using mask sizes set as a factor proportional to the standard deviations in the model. Because of the sparse nature and relatively low data rate of the sonar, this idea could be used by the interpretation process to form connected clusters of cells, i.e. grouping echoes into clusters corresponding to objects. Note however that the $3 \mathrm{D}$ mask sizes must be chosen carefully in order not to be too resource demanding.

\subsection{Data organization}

Based on the previous discussion it is natural to choose a spherical coordinate frame for the grid. This reflects the properties of the sonar sensor in a very direct way, i.e. beam orientation and range readings. In this framework it is straightforward to directly allocate a sonar echo to the corresponding place in 3D space. Furthermore it allows a cell geometry reflecting the sonar geometric properties. As an example some of the 


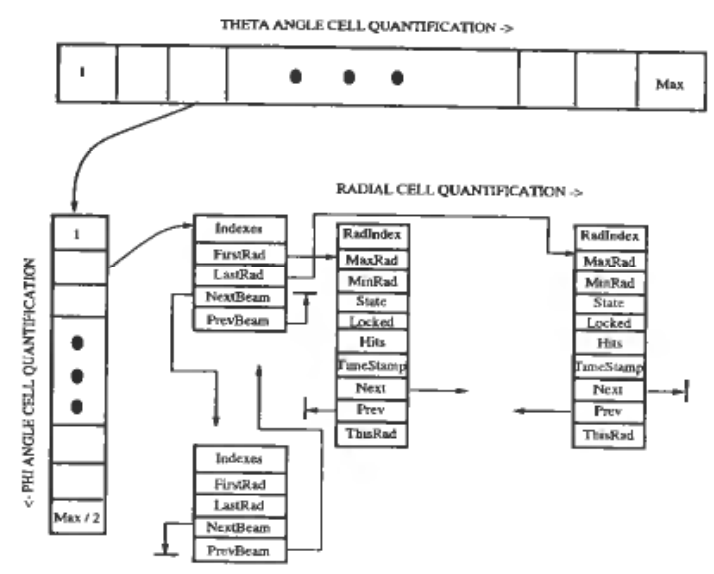

Figure 5. 3D spherical grid data structure.

properties of the Simrad EM 1000 multibeam sonar working in shallow mode are quoted below (Berge, 1993a):

- 60 beams.

- 50 degrees sector width.

- 2.5 degrees beam spacing.

- Accuracy $0.3 \%$ of water depth (surface vessel mounted sonar) or maximum $15 \mathrm{~cm}$ resolution.

In a spherical grid it is quite feasible to let cell geometry reflect these properties. For short range radial cell size could be equal to maximum resolution, whereas further away from the AUV, radial cell size increases as a geometric series. As such the grid could be viewed as spherical layers of increasing thickness. For a fixed range, this approach will still give a fixed number of cells in the radial direction. Simulations have indicated that such a grid is feasible, even in a realtime perspective (grid update rate keeps up with sensor data rate). Implementing this grid, only the theta and phi angles (standard spherical coordinate definition, refer to figure 2) were quantified into 'buckets', allowing flexibility in the range parameter. It would be useful to be able to store grids with different resolutions allowing 'zooming' effects, specially when searching for echo clusters. Figure 5 shows a feasible organization of the grid data structure. Grid cells are organized by two doubly linked lists, one ordered by beam indexes (the two spherical angles) and the other by range. For each beam there is a list of cells sorted by distance. As closer echoes will arrive to the AUV first, there should be little overhead filling in the cells in the correct order. To allow fast 'look-up' of beam directions with echoes, two hash-tables are included in the structure. Note that the phi table only exist for those theta quantifications where echoes were found, minimizing memory storage and allowing fast checking of 'empty' theta and phi directions. The links between beam cells is ordered as the hashtables indicate: increasing phi for each theta angle quantification. This list allows fast data processing of only the cells actually stored, without traversing all elements of the hashtables.

For each cell several features are stored: Radial limits, probability state, total number of echoes in this cell, time elapsed since first and last echo and a binary 'lock' parameter. This last parameter is used to lock the cell state when sufficient items of evidence exists to claim it to be occupied. It could also be used to give cells a priori 
and unchangeable states, for example when placing a priori known (or geometrical results from the modeling process) objects in the grid. Furthermore another list between cells at similar radial distance could be implemented (utilizing the ThisRad pointers), a list specially useful for RCD (Leonard, Durrant-White and Cox 1992) or Tangential Cluster (Mandelbaum and Mintz 1994) based algorithms.

Another interesting question is grid orientation. It would be advantageous to keep the orientation fixed when referring to the location of a priori known objects using some sort of area maps. By using information from other parts of the onboard AUV expert system such as the INS and dynamics control system (including current and disturbance measurements) the grid could (as an example) be fixed with the $x$-axis in the East direction and $z$ positive upwards. Another possibility is to define a 'course-sensitive grid' using the current course as the $y$-direction (refer to figure 2).

The symmetric properties of the spherical grid allows flexibility in orientation. Notice how straightforward cells could be rotated by only updating the theta and phi pointers. This grid is implemented as a dynamical pointer structure in the $\mathrm{C}$ programming language, allowing fast creation and deletion of multiple grids. The presented structure is however not intended to be final, but should be regarded as a reflection of current ideas.

\subsection{Data visualization}

Simulation and visualization are useful and necessary tools for AUV design (Brutzmann, Kanayama and Zyda 1992). At the Department of Engineering Cybernetics, there has been a similar research project called MOBATEL (Volden and Balchen 1993), which also emphasize the use of powerful graphical workstations. Graphical visualization is specially important when working in 3D.

So far a visualization tool has been implemented using Matlab to inspect the 3D grid resulting from different algorithms. This tool allows inspection from arbitrary viewing directions including zooming. The grid is presented as a 3D grey level image, the grey level reflecting cell state. For a illustration of how such as 3D sonar image could look refer to Fig. 6.

Figure 6 shows (top to bottom) a view of the 2D cross-section and a 3D path within this virtual geometry (noisy sinusoidal movement in the $z$ direction added to a 2D path in the $x y$-plane). The first ten 'sonar beam locations' along the path are enumerated. The second picture is the resulting grid in position 10 seen from above, keeping track of sonar scans taken along the path. The four outer corner points of each cell is included to make small cells visible. The third picture is a 3D view of the same grid, illustrating its real structure. The dotted lines in are spaced 30 degrees apart in the $x y$-plane centred at the current position. Note that cell sizes vary with distance, both in radial and angular directions.

\section{Conclusion and further work}

The proposed 3D grid is a feasible data structure for realtime sonar echo ranging that should be investigated further. Several interesting questions still remain unanswered:

- Grid updating policies, cell state calculations based on new measurements, elapsed time and number of echoes, compensating for grid cell volume differences. 

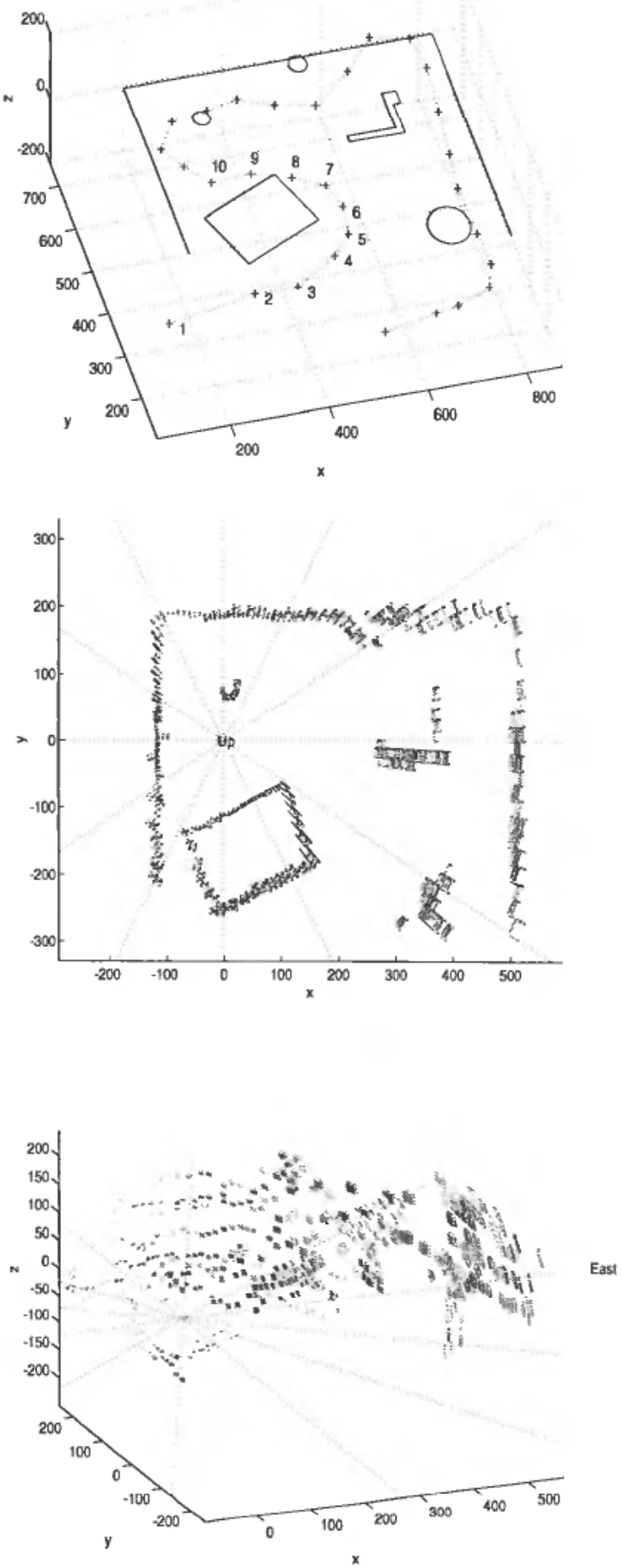

Figure 6. Path in geometric model and resulting 3D grid. 
- Algorithms for utilizing the grid: Path planning, obstacle avoidance, active vision approaches.

- Realtime experiments on sonar data, investigation of how to handle multipath propagation effects, false and spurious echoes within this framework.

Currently the research is focused on a hardware design for coupling the range data from a SS150 WesMar $6.5^{\circ} \times 6.5^{\circ}$ beamwidth sweep sonar with software implementing the presented grid framework. This sonar is mounted on a surface vessel, but as its transponder is controlled by two brushless DC motors in the theta and phi direction it seems to be quite suitable for interesting experiments within the presented framework.

\section{ACKNOWLEDGEMENTS}

The authors wish to thank Donald P. Brutzmann, Naval Postgraduate School, USA and Geir Mathisen, SINTEF Automatic Control, Norway, for their helpful support of information and software regarding their research. This research was supported by NorFA (grant $94.30 .150-O$ ) and Norges Forskningsråd, greatly appreciated by the authors.

\section{REFERENCES}

BAJCSY, R. (1988). Active perception, Proceedings of the IEEE, 76 (8), pp. 996-1005.

Bergem, O., ANDERSEN, C. S. and Christensen, H. I. (1993). Using match uncertainty in the Kalman filter for a sonar based positioning system. Proceedings of the 8th Scandinavian Conference in Image Analysis (SCIA), Troms $\emptyset$, Norway, May 25-28, pp. 405410 , Published by NOBIM (Norwegian Society for Image Processing and Pattern Recognition).

Bergem, O. (1993a). Bathymetric Navigation of Autonomous Underwater Vehicles using a Multibeam Sonar and a Kalman Filter with relative Measurement Covariance Matrices. Dr Scient thesis, University of Trondheim, Norway.

BERGEM, O. (1993b). A multibeam sonar based positioning system for an AUV. Proceedings of the 8th International Symposium on Unmanned Untethered Submersible Technology, USA. September 27-29, pp. 291-299. Published by Autonomous Undersea Institute, Portsmouth, NH.

BrutZMANN, D. P., COMPTON, M. A. and Kanayama, Y.(1992). Autonomous sonar classification using expert systems. Proceedings of the IEEE Oceanic Engineering Society OCEANS 92 Conference, Newport, USA. October 26-29, pp. 554-559, IEEE.

BrutzmanN, D, P., Kanayama, Y. and ZYDA, M. J. (1992). Integrated simulation for rapid development of autonomous underwater vehicles. Proceedings of the IEEE Oceanic Engineering Society Autonomous Underwater Vehicle (AUV) 92 Conference, Washington DC., USA. June 4-5, pp. 3-10, IEEE.

DURRANT-WhYTE, H. F. (1988). Sensor models and multisensor integration. The International Journal of Robotics Research, 7, No. 6, pp. 73-89.

Elfes, A. (1990). Occupancy Grids: A stochastic spatial representation for active robot perception. Proceedings of the Sixth Conference on Uncertainty in AI, July 1990. Morgan Kaufmann, Publishers.

ELFES, A. (1991). Dynamic control of robot perception using stochastic spatial models. Proceedings of the International Workshop on Information Processing in Mobile Robots. March 1991. Springer Verlag, New York.

Fossen, T. I. (1991). Nonlinear Modelling and Control of Underwater Vehicles. Dr. Ing thesis, The Norwegian Institute of Technology.

Leonard, J. J., Durrant-Whyte, H. F. and CoX, I. J. (1992). Dynamic map building for an autonomous mobile robot. The International Journal of Robotics Research, 11, No. 4.

Mandelbaum, R. and MinTZ, M. (1994). Sonar signal processing using tangent clusters. Proceedings of the IEEE Oceanic Engineering Society OCEANS 94 conference, September 1994, Brest, France.

MATHISEN, G. (1993). A system for mapping of a partly structured environment for an AUV. Proceedings of the 8th International Symposium on Unmanned Untethered Submersible. 
Technology. September 27-29, pp. 329-345. Published by Autonomous Undersea Institute, Portsmouth, NH.

MA'TTHIES, L. and ELFES, A. (1988). Integration of sonar and stereo range data using a grid-based representation. Proceedings of the IEEE International Conference on Robotics and Automation, pp. 727-733.

Moran, B. A., Leonard, J. J. and Chryssostomidis, C. (1993). Geometric shape from sonar ranging. Proceedings of the 8th International Symposium on Unmanned Untethered Submersible Technology, September 27-29, pp. 370-383. Published by Autonomous Undersea Institute, Portsmouth, NH.

OL'SHEVSKII, V. V. (1978). Statistical Methods in Sonar (Consultants Bureau, New York (English edition)).

Smith, R., Self, M. and Cheeseman, P. (1987). A stochastic map for uncertain spatial relationships. Proceedings of the 1987 International Symposium on Robotics Research, pp. 323-330, MIT Press.

Urick, R. J. (1983). Principles of Underwater Sound. (McGraw-Hill (3rd edition)).

VOLDEN, R. and BALCHEN, J. (1993). Determining 3-D Object coordinates from a sequence of 2-D images in modelling ROV environments. Proceedings of the 8th International Symposium on Unmanned Untethered Submersible Technology, September 27-29, pp. 359-369. Published by Autonomous Undersea Institute, Portsmouth, NH. 